$\begin{array}{cl}\text { PRAMANA } & \text { (C) Indian Academy of Sciences } \\ \begin{array}{c}\text { journal of } \\ \text { physics }\end{array} & \begin{array}{l}\text { Vol. } 65, \text { No. } 4 \\ \text { October } 2005 \\ \text { pp. } 549-564\end{array}\end{array}$

\title{
Soft chemical routes to semiconductor nanostructures
}

\author{
UJJAL K GAUTAM $^{1,2}$, KRIPASINDHU SARDAR $^{1,2}$, F L DEEPAK $^{1}$ \\ and $\mathrm{C}$ N R RAO ${ }^{1,2, *}$ \\ ${ }^{1}$ Chemistry and Physics of Materials Unit and CSIR Centre of Excellence in Chemistry, \\ Jawaharlal Nehru Centre for Advanced Scientific Research, Jakkur P.O., \\ Bangalore 560 064, India \\ ${ }^{2}$ Solid State and Structural Chemistry Unit, Indian Institute of Science, \\ Bangalore 560 012, India \\ ${ }^{*}$ Author for correspondence. E-mail: cnrrao@jncasr.ac.in
}

\begin{abstract}
Soft chemistry has emerged as an important means of generating nanocrystals, nanowires and other nanostructures of semiconducting materials. We describe the synthesis of CdS and other metal chalcogenide nanocrystals by a solvothermal route. We also describe the synthesis of nanocrystals of AlN, GaN and InN by the reaction of hexamethyldisilazane with the corresponding metal chloride or metal cupferronate under solvothermal conditions. Nanowires of Se and Te have been obtained by a self-seeding solution-based method. A single source precursor based on urea complexes of metal chlorides gives rise to metal nitride nanocrystals, nanowires and nanotubes. The liquidliquid interface provides an excellent medium for preparing single-crystalline films of metal chalcogenides.
\end{abstract}

Keywords. Soft-chemical route; semiconductor nanocrystal; solvothermal; liquid-liquid interface.

PACS Nos 81.16.-c; 81.07.-b; 81.07.Bc

\section{Introduction}

Nanocrystals, nanowires and nanotubes of a large number of inorganic materials have been synthesized and characterized in the last five years [1-5]. Some of the nanomaterials exhibit properties of potential technological value. This is particularly true for nanostructures of semiconducting materials such as metal chalcogenides and nitrides. In view of the importance of these materials, it is necessary to find better methods of synthesis which yield crystalline nanostructures of high quality. We have been exploring several soft-chemical methods in this direction. In this article, we give a brief report of the successful soft-chemistry methods for the preparation of nanostructures of metal chalcogenides such as CdS, CdSe and $\mathrm{CuS}$ as well as group-13 nitrides such as AlN, GaN and InN. In addition, we report the results of our recent studies employing the liquid-liquid interface, whereby we 
Ujjal K Gautam et al

are able to prepare extended ultra-thin single-crystalline films and nanocrystals of metal chalcogenides.

\section{Semiconductor nanocrystals}

\subsection{Metal chalcogenide nanocrystals}

We have been able to prepare size-tunable, organic-soluble CdS nanocrystals by employing a novel solvothermal technique [6]. In this method, cadmium stearate $\left[\mathrm{Cd}(\mathrm{St})_{2}\right]$ is reacted with sulfur and tetralin in toluene solvent, wherein tetralin forms naphthalene giving out hydrogen sulfide. The $\mathrm{H}_{2} \mathrm{~S}$ generated in situ by the hydrogen reduction of sulfur, reacts with $\mathrm{Cd}(\mathrm{St})_{2}$ to yield $\mathrm{CdS}$ nanocrystals. By employing a suitable capping agent such as trioctylphosphineoxide (TOPO) or dodecanethiol, the growth of the resulting phase can be arrested giving rise to toluenesoluble monodisperse CdS nanocrystals. In a typical reaction for the preparation of dodecanethiol-capped CdS nanocrystals, $0.46 \mathrm{~g}(0.68 \mathrm{mmol})$ of $\mathrm{Cd}(\mathrm{St})_{2}$ was mixed with $0.022 \mathrm{~g}(0.68 \mathrm{mmol})$ of sulfur, $0.1 \mathrm{~g}(0.75 \mathrm{mmol})$ of tetralin and $0.66 \mathrm{~g}(3.3$ mmol) of dodecanethiol in $45 \mathrm{ml}$ toluene. The reactants were sealed in a stainless steel $70 \mathrm{ml}$ teflon-lined autoclave and placed in an air oven which was preheated to $220^{\circ} \mathrm{C}$ for $12 \mathrm{~h}$. TOPO-capped CdS nanocrystals were prepared in a similar fashion. Figure 1a shows a TEM image of the TOPO-capped CdS nanocrystals. The particles are fairly spherical and monodisperse with a mean diameter of $4 \mathrm{~nm}$ and a standard deviation of $0.4 \mathrm{~nm}$ ( $10 \%$ of the mean). We show the histogram of the particle size distribution as well as a high resolution electron microscope (HREM) image of a single nanocrystal as insets in figure 1a. The lattice fringes observed in the HREM images indicate a high degree of crystallinity. When the CdS particles were capped with dodecanethiol, we obtained larger, $10 \mathrm{~nm}$ nanocrystals which were insoluble, besides soluble $5 \mathrm{~nm}$ particles. Figure $1 \mathrm{~b}$ shows the $5 \mathrm{~nm}$ thiolcapped CdS nanocrystals. The selected area electron diffraction (SAED) pattern of the nanocrystals is shown as the inset. The calculated $d$ values from the SAED pattern match with those expected for the space group F-43m. It is interesting that the nanocrystals crystallize in the cubic structure rather than in the stable hexagonal structure. In figure $1 \mathrm{c}$, we show the powder X-ray diffraction patterns (XRD) of the 4, 5 and the $10 \mathrm{~nm}$ CdS nanocrystals. To confirm the structure, a XRD pattern was simulated for the cubic CdS crystal structure (broken curve in figure 1c) which compares well with the observed patterns. The UV-vis absorption spectra of the nanocrystals show a blue-shift of the absorption edge indicating quantum confinement (figure 1d). The TOPO-capped $4 \mathrm{~nm}$ nanocrystals exhibit a well-defined absorption feature at $455 \mathrm{~nm}$. This is considerably blue-shifted relative to the bulk value of $515 \mathrm{~nm}[7]$.

This method can be extended to prepare other chalcogenide nanocrystals as well. Thus, we have synthesized toluene-soluble $3 \mathrm{~nm}$ CdSe nanocrystals [8] by reacting $\mathrm{Cd}(\mathrm{St})_{2}$ with selenium instead of sulfur. The UV-vis absorption spectrum of the 3 $\mathrm{nm}$ nanocrystals shows an absorption band at $514 \mathrm{~nm}$ (bulk, $680 \mathrm{~nm}$ ), consistent with quantum confinement. We have prepared $\mathrm{PbS}$ and $\mathrm{PbSe}$ nanocrystals also by the same method [9]. Besides good yields of the nanocrystals, this method has certain advantages over other techniques. The reaction is carried out in a closed 

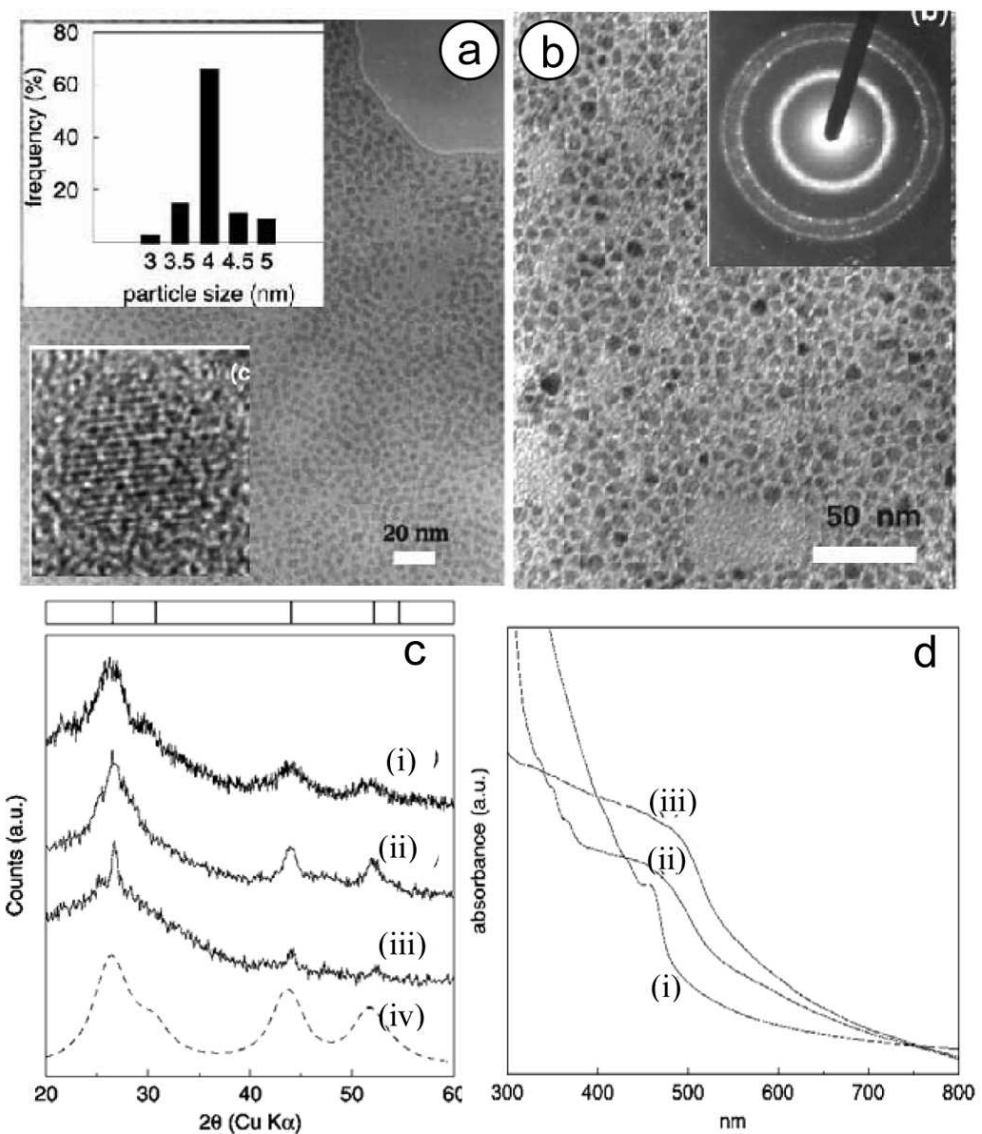

Figure 1. TEM image of (a) TOPO-capped CdS nanocrystals (insets show the size distribution of the particles and HREM image of a nanocrystal), (b) soluble dodecanethiol-capped CdS nanocrystals. Inset shows the corresponding SAED pattern. (c) Powder XRD patterns of (i) $4 \mathrm{~nm}$ TOPO-capped, (ii) 5 $\mathrm{nm}$ soluble and (iii) $10 \mathrm{~nm}$ insoluble dodecanethiol-capped CdS nanocrystals. (iv) Shows the simulated XRD pattern for the $4 \mathrm{~nm}$ particles. Vertical lines at the top represent expected peak positions. (d) UV-vis absorption spectra of the (i) $4 \mathrm{~nm}$ (ii) $5 \mathrm{~nm}$ and (iii) $10 \mathrm{~nm}$ CdS nanocrystals.

vessel and inert conditions are, therefore, not required. Since the nanocrystals are toluene-soluble, they can be size selectively precipitated using a polar solvent leading to monodispersity. Unlike in room-temperature synthesis, we obtain highlycrystalline nanoparticles.

\subsection{Group-13 nitride nanocrystals: AlN, GaN and InN}

We have developed two new solvothermal routes to prepare nanocrystals of hexagonal, group-13 nitrides, namely AlN, GaN and InN. One route employs the reaction 


\section{Ujjal K Gautam et al}

between 1,1,1,3,3,3-hexamethyldisilazane (HMDS) $\left[\left(\mathrm{CH}_{3}\right)_{3} \mathrm{SiNHSi}\left(\mathrm{CH}_{3}\right)_{3}\right]$ with the organometallic precursor, metal cupferronate, $\mathrm{M}(\mathrm{cup})_{3},\left[\mathrm{M}\left(\mathrm{C}_{6} \mathrm{H}_{5} \mathrm{~N}_{2} \mathrm{O}_{2}\right)_{3}\right](\mathrm{M}=\mathrm{Al}$, $\mathrm{Ga}, \mathrm{In})$ and the other with the metal trichloride, $\mathrm{MCl}_{3}$. All the reactions are carried out in anhydrous toluene in the presence or absence of surfactants like cetyl trimethyl ammonium bromide (CTAB) or hexadecyl amine (HDA) inside a stainless steel autoclave (swagelok). Details of the sample preparation can be found elsewhere [10-12].

The solvothermal reaction of $\mathrm{M}(\operatorname{cup})_{3}(\mathrm{M}=\mathrm{Al}$, Ga and $\mathrm{In})$ with HMDS in toluene yields the nitride nanocrystals in the $250-450^{\circ} \mathrm{C}$ temperature range. In figure 2, we show typical TEM images of the as-synthesized nanocrystals along with the particle size distributions. In this procedure, it was possible to obtain nanocrystals with average diameters of $\sim 10 \mathrm{~nm}$ for $\mathrm{AlN}, \sim 15 \mathrm{~nm}$ for $\mathrm{InN}$ and as low as $\sim 4 \mathrm{~nm}$ for GaN. The nanocrystals are single crystalline as confirmed by the observation of lattice fringes in the HREM image (see insets in figure 2). The cupferron precursor under solvothermal conditions first decomposes to give an active oxide species $\mathrm{MO}_{1.5}$ which then reacts with HMDS to form the metal nitride and hexamethyldisiloxane. Reaction of $\mathrm{MCl}_{3}$ with HMDS readily gives the nitride nanocrystals with the elimination of $\mathrm{Me}_{3} \mathrm{SiCl}$. Typical TEM images of the nitride nanocrystals obtained by this procedure are shown in figure 3 . The procedure has enabled us to obtain GaN nanocrystals with an average diameter of $\sim 2.5 \mathrm{~nm}$ with a narrow size distribution (see inset in figure 3b). PL spectra of the GaN nanocrystals with average diameters of $\sim 2.5, \sim 4$ and $\sim 12 \mathrm{~nm}$, prepared by us show interesting features (figure 4 ). While the $\sim 12 \mathrm{~nm}$ nanocrystals show a broad band around $375 \mathrm{~nm}$ at an excitation wavelength of $250 \mathrm{~nm}$, the $\sim 4 \mathrm{~nm}$ nanocrystals show an additional intense band around $315 \mathrm{~nm}$, which we believe characterizes quantum confinement. The $375 \mathrm{~nm}$ band is insensitive to particle size [13]. The lower wavelength band is, however, sensitive to size. Thus, GaN nanocrystals of $\sim 2.5 \mathrm{~nm}$ diameter (shown in the TEM image in figure 3b) exhibit bands at 375 and $265 \mathrm{~nm}$ at an excitation wavelength of $235 \mathrm{~nm}$. The $265 \mathrm{~nm}$ band is the blue-shifted band corresponding to the $315 \mathrm{~nm}$ band exhibited by the $\sim 4 \mathrm{~nm}$ particles. The absorption spectra of the nanocrystals of InN gave a band centered around $0.8 \mathrm{eV}$ which is its intrinsic characteristic, rather than the $1.9 \mathrm{eV}$ band reported earlier $[14,15]$.

\section{Nanowires and nanotubes}

\subsection{Se and Te nanowires}

Selenium shows interesting properties such as high photoconductivity and catalytic activity and has commercial use in photocopiers and rectifiers [16]. Nanorods of t-selenium have been synthesized by a simple solution-based method [17] wherein selenium powder is first reacted with $\mathrm{NaBH}_{4}$ to yield NaHSe [18] in aqueous solution to obtain amorphous selenium. In a typical room temperature reaction, $0.025 \mathrm{~g}$ $(0.32 \mathrm{mmol})$ of selenium was mixed with $20 \mathrm{ml}$ of de-ionized water in a three-necked round bottom flask. $0.030 \mathrm{~g}(0.81 \mathrm{mmol})$ of $\mathrm{NaBH}_{4}$ was added to this mixture and the flask was purged with nitrogen gas in order to create an inert atmosphere. The 
Soft chemical routes to semiconductor nanostructures

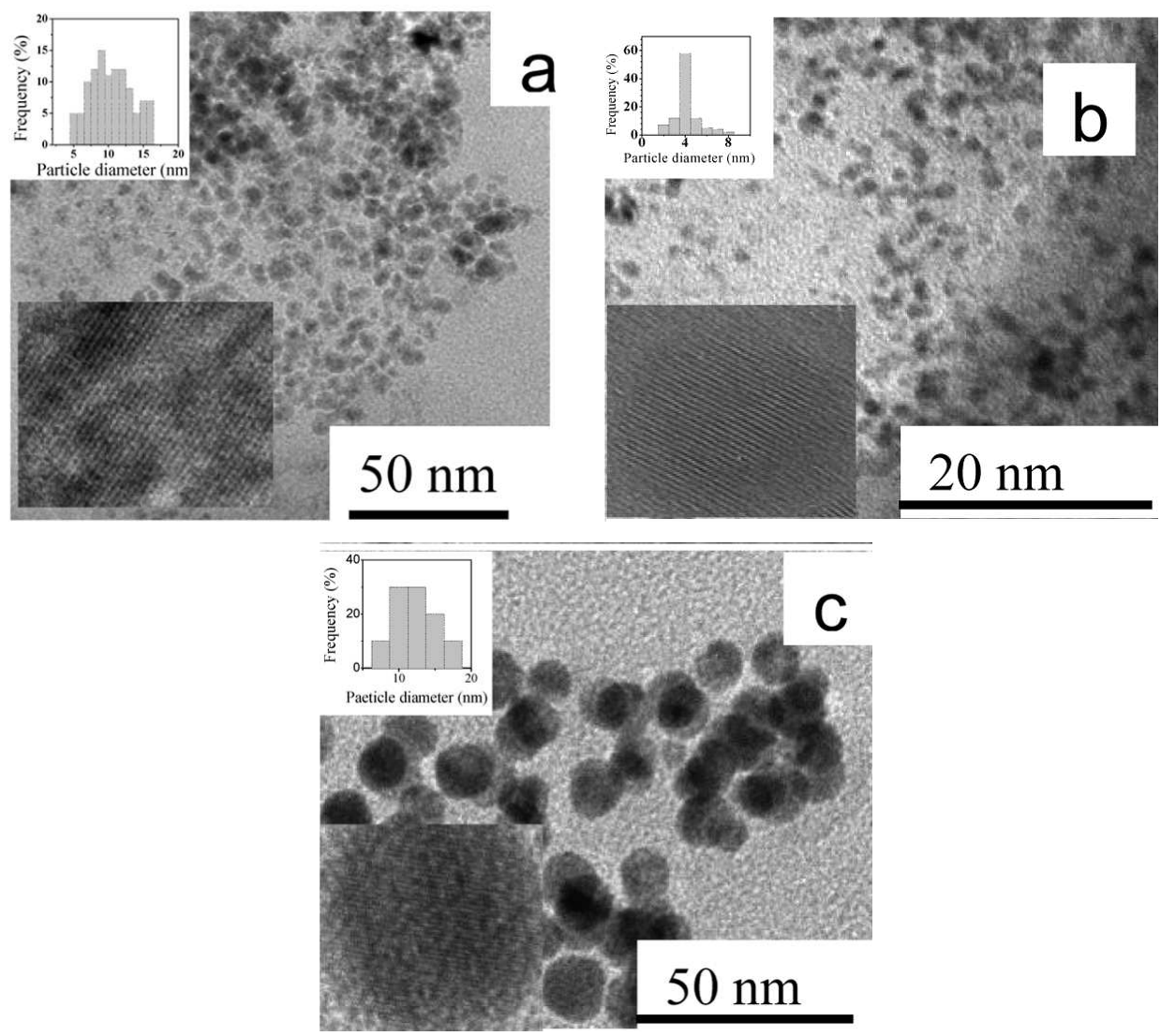

Figure 2. TEM images of (a) AlN, (b) GaN, (c) InN nanocrystals obtained from metal cupferronate and HMDS. Insets in (a), (b) and (c) show HREM images of the nanocrystals (bottom left) and size distributions (top left).

selenium dissolves in water in 60 min giving rise to a clear colourless solution. The reaction mechanisms involved are:

$$
\begin{aligned}
& 4 \mathrm{NaBH}_{4}+2 \mathrm{Se}+7 \mathrm{H}_{2} \mathrm{O} \rightarrow 2 \mathrm{NaHSe}+\mathrm{Na}_{2} \mathrm{~B}_{4} \mathrm{O}_{7}+14 \mathrm{H}_{2}, \\
& \mathrm{Na}_{2} \mathrm{~B}_{4} \mathrm{O}_{7}+2 \mathrm{NaHSe}+2 \mathrm{Se}+5 \mathrm{H}_{2} \mathrm{O} \rightarrow 2 \mathrm{Na}_{2} \mathrm{Se}_{2}+4 \mathrm{H}_{3} \mathrm{BO}_{3}, \\
& \mathrm{Na}_{2} \mathrm{Se}_{2}+2 \mathrm{H}_{2} \mathrm{O} \rightarrow 2 \mathrm{a}-\mathrm{Se}+2 \mathrm{NaOH}+\mathrm{H}_{2}, \\
& \text { a-Se } \rightarrow \text { t-Se. }
\end{aligned}
$$

The selenide ion acts as the source of Se in this method while the methods reported in the literature generally use the oxyanions with a positive charge on Se as the source. The nascent selenium produced in step (3) imparts a wine red colour to the aqueous solution. On standing for a few hours, the solution transforms into amorphous Se in colloidal form. A small portion of the dissolved selenium precipitates as t-Se nanoparticles which act as nuclei to form one-dimensional nanorods. 

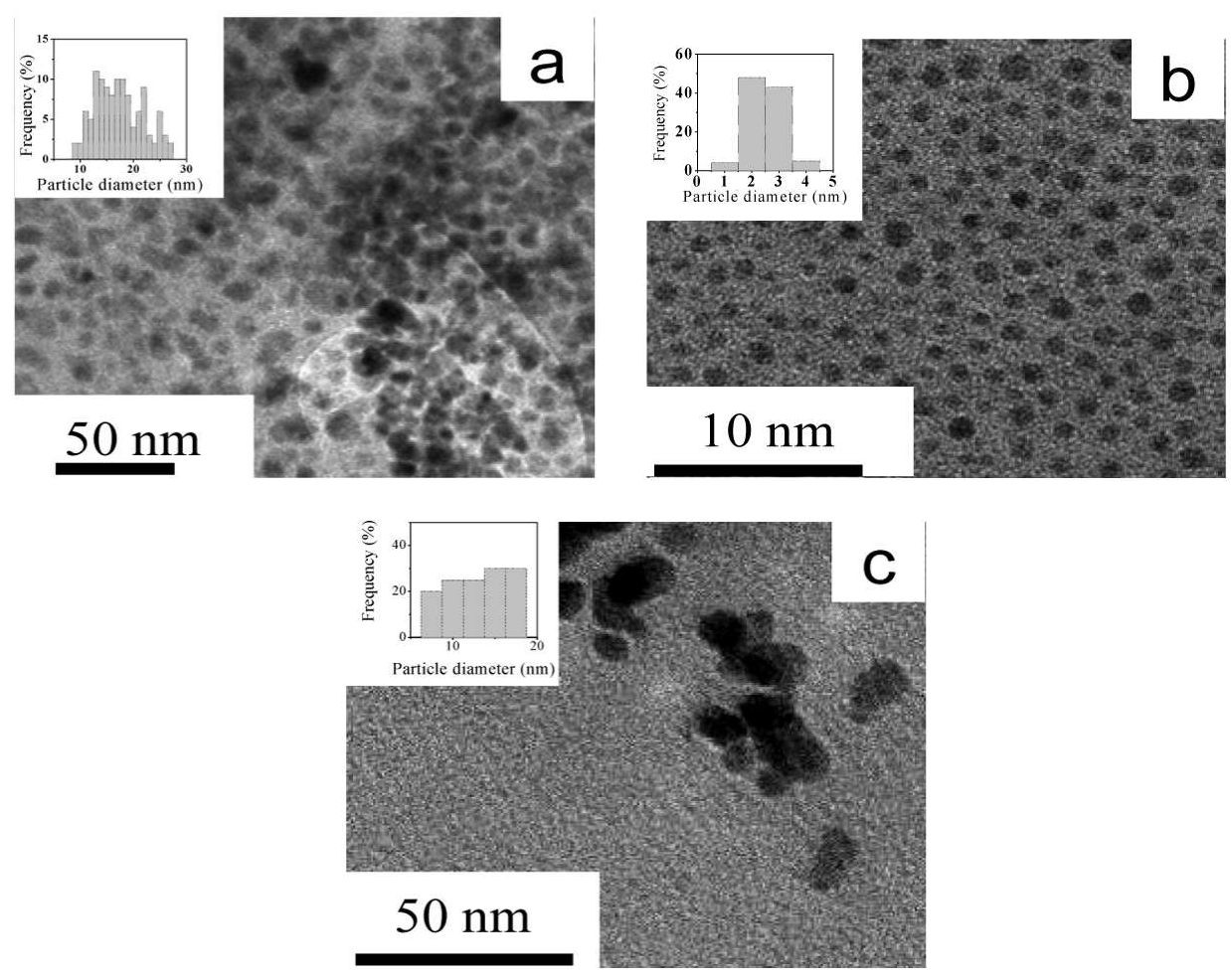

Figure 3. TEM images of (a) AlN, (b) GaN, (c) InN nanocrystals obtained from metal chlorides and HMDS. Insets in (a), (b) and (c) show size distribution.

The amorphous Se colloid dissolves slowly as the nanorods increase in length. In the process, the red colour of the solution fades away and dark selenium nanorods settle down. In figure 5a, we show a SEM image of the nanorods formed at $30^{\circ} \mathrm{C}$ after $96 \mathrm{~h}$. The diameter of the rods is around $150 \mathrm{~nm}$. The rods grow in length with time but the diameters remain almost the same. The HREM image shown in figure $5 \mathrm{~b}$ reveals that the rods are single crystalline, devoid of crystallographic defects. The lattice spacings of $4.9 \AA$ in the HREM image along the growth direction corresponds to the separation between the $(001)$ planes, showing thereby that the growth direction is along the $c$-axis. We obtained shorter nanorods when the reaction mixture was quenched at $0^{\circ} \mathrm{C}$ for $96 \mathrm{~h}$. The diameter of the nanorods could be decreased by diluting the reaction mixture. Thus, $20 \mathrm{~nm}$ nanorods were obtained when the reaction mixture was diluted four times. These nanorods assemble in bundles. We observed an interesting morphology of t-Se when the synthesis was carried out under hydrothermal conditions or in the presence of a capping agent. Use of polyvinylpyrrolidone leads to a flowery morphology (figure 5c). The reaction carried out under solvothermal conditions gives tubular structures, with a wall thickness of the order of $100 \mathrm{~nm}$ (figure 5d). 
Soft chemical routes to semiconductor nanostructures

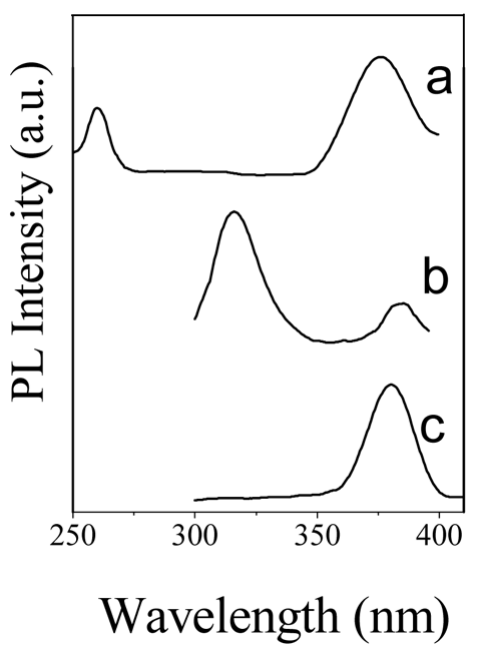

Figure 4. PL spectra of GaN nanocrystals of different sizes: (a) $\sim 2.5 \mathrm{~nm}$ (excitation at $235 \mathrm{~nm}$ ), (b) and (c) $\sim 4 \mathrm{~nm}$ and $\sim 12 \mathrm{~nm}$ (excitation wavelength $250 \mathrm{~nm})$.

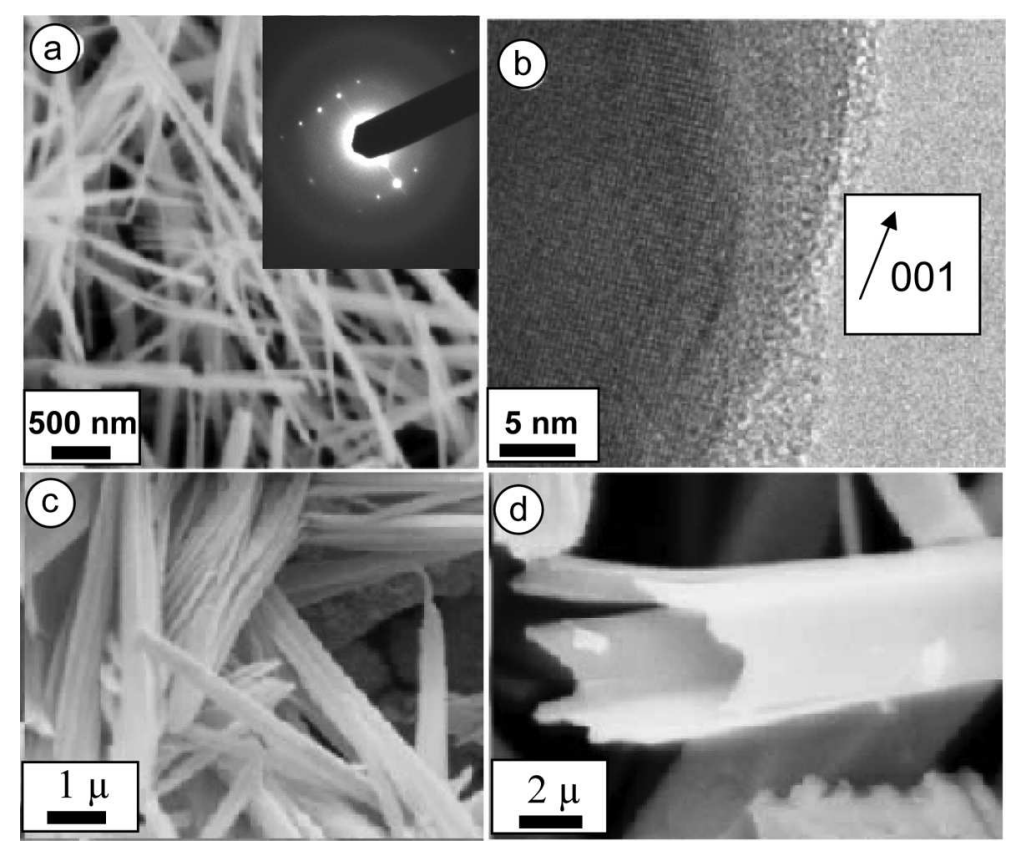

Figure 5. (a) SEM image of t-Se nanorods after $96 \mathrm{~h}$. Inset shows a SAED pattern obtained from one of the rods. (b) HREM image of one of the nanorods. (c) SEM image of PVP-capped selenium nanoflowers. (d) Se tubular structure obtained under hydrothermal conditions. 


\section{Ujjal K Gautam et al}

Extending the above strategy, we could obtain Te nanorods, nanowires, nanobelts and junction nanostructures as well [19]. The reaction of Te $(0.030 \mathrm{~g})$ with $\mathrm{NaBH}_{4}$ $(0.050 \mathrm{~g})$ in aqueous medium gave uniform nanowires of $100-300 \mathrm{~nm}$ diameter with lengths of approximately $10 \mu \mathrm{m}$ (figure $6 \mathrm{a}$ ). The nanorods grow with time and become smoother and monodisperse as time passes. Figure 6b shows a HREM image of one of the nanorods. The fringe spacing of $5.8 \AA$ observed in the image corresponds to the separation between the $(001)$ planes of hexagonal Te. The image reveals that the growth of the nanorods is perpendicular to this plane and hence along the $\langle 001\rangle$ direction. The SAED pattern of the rods (see inset in figure $6 \mathrm{~b}$ ) confirms the single crystalline nature of the rods. The diffraction spots could be indexed on the basis of the hexagonal structure of Te. Nanorods of smaller dimensions were obtained at lower initial concentration of the reactants. For example, when a reaction mixture of $0.0300 \mathrm{~g}$ of $\mathrm{Te}$ powder and $0.0500 \mathrm{~g}$ of $\mathrm{NaBH}_{4}$ was diluted with $150 \mathrm{ml}$ of hot de-ionized water, just after the dissolution of the $\mathrm{Te}$ powder, we obtained uniform nanorods of $20 \mathrm{~nm}$ diameter and $400 \mathrm{~nm}$ length. The nanowires show interesting optical absorption and luminescent properties. Unlike
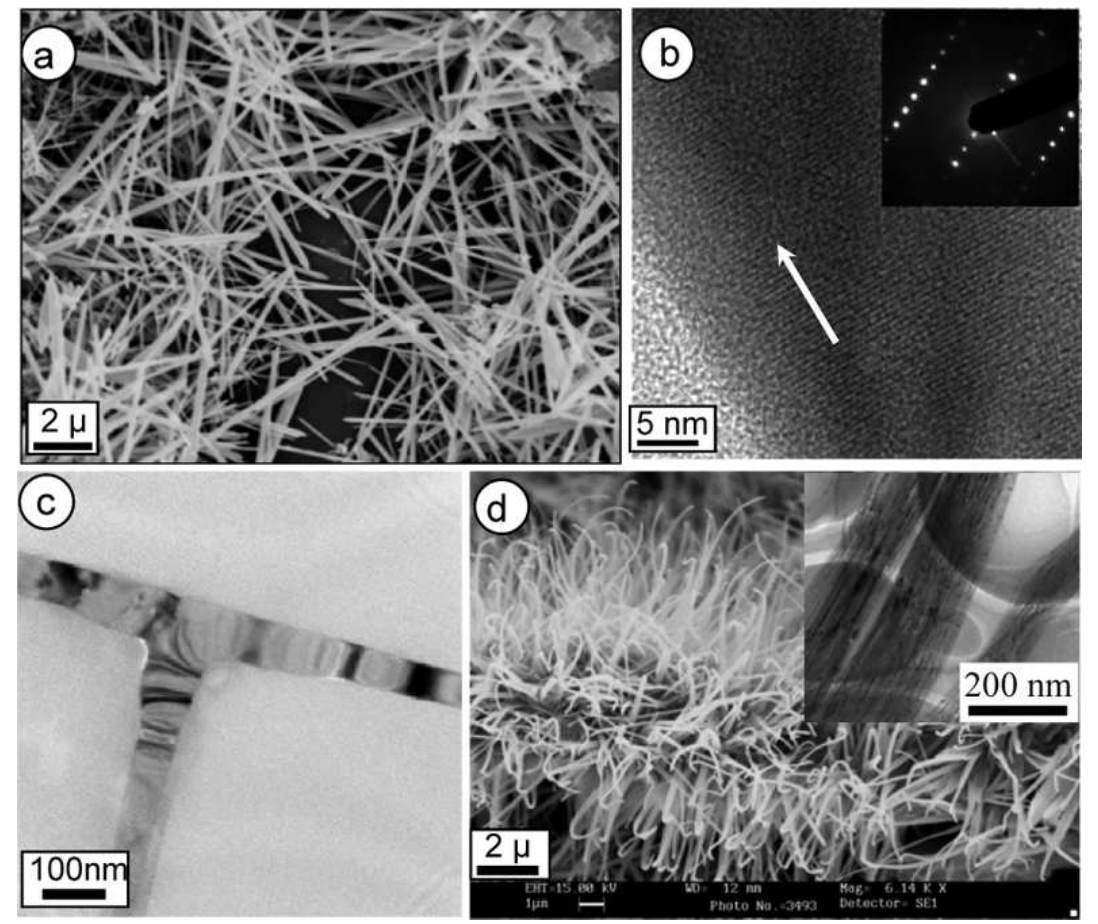

Figure 6. (a) SEM image of Te nanorods obtained from the reaction of 0.03 $\mathrm{g}$ of Te with $\mathrm{NaBH}_{4}$ in $20 \mathrm{ml}$ water. (b) HREM image of one of the nanorods showing (0 01 ) planes of hexagonal Te (inset shows the corresponding SAED pattern). (c) A nanobelt of Te containing a T-junction obtained by hydrothermal synthesis. (d) Low magnification SEM image of aligned Te nanobelts. The inset is a TEM image showing a collection of aligned nanobelts. 
solution-phase synthesis, where uniform nanorods were obtained, the same synthetic procedure under hydrothermal conditions at $150^{\circ} \mathrm{C}$ yielded crystalline Te nanobelts of thickness 5-10 nm thickness. Their tendency to form branches and junctions as shown in figure $6 \mathrm{c}$ is quite interesting. When the reaction was carried out in the presence of the surfactant sodium dodecylbenzenesulfonate we obtained aligned nanobelts. Figure 6d shows a low-resolution SEM image of aligned nanobelts. Interestingly, each of these 1D nanostructures, on TEM investigation, revealed to be bundles of much smaller aligned nanobelts (shown as inset in figure 6d).

\subsection{Group-13 nitrides}

InN: The reaction of indium acetate with HMDS in the absence of any solvent at $300^{\circ} \mathrm{C}$ gives interesting nanostructures consisting of both nanowires and nanotubes with yields of $40 \%$ and $60 \%$ respectively. The XRD pattern of these nanostructures was characteristic of wurtzite InN. The SEM image in figure 7 a shows the presence of a large number of nanowires and nanotubes. The nanowires have diameters

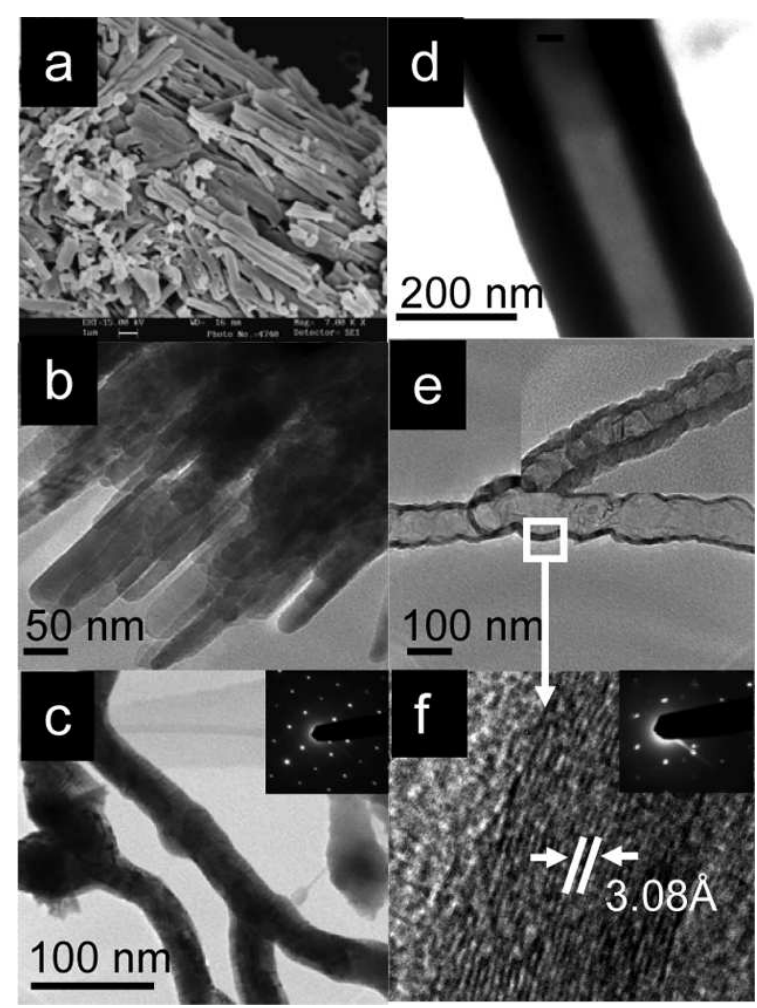

Figure 7. InN nanowires and nanotubes: (a) SEM image; (b), (c) TEM images of nanowires; (d), (e) TEM images of nanotubes; (f) HREM image of a wall of a nanotube. Insets in (c) and (f) show the SAED patterns on a nanowire and nanotube respectively. 


\section{Ujjal K Gautam et al}

in the 50-100 nm range, with lengths extending up to a micron as seen in the low-resolution TEM images in figure $7 \mathrm{~b}$. We have found the presence of a few Yjunction nanowires as well (figure $7 \mathrm{c}$ ). The nanowires are single crystalline with the Bragg spots corresponding to the (100), (1 01$)$ and (0 02 2) planes in the SAED pattern (see inset of figure 7c). HREM images of the nanowires gave interplanar spacings of $\sim 2.7 \AA$ corresponding to the (101) plane of the wurtzite structure, with the growth direction of the nanowires being parallel to the (101) plane. In figures $7 \mathrm{~d}$ and $7 \mathrm{e}$, we show the TEM images of the InN nanotubes. They show an interlayer spacing of $3.08 \AA$ corresponding to the (100) planes as revealed in the HREM image in figure $7 \mathrm{f}$. This spacing is distinctly lower than the interlayer spacing of $3.4 \AA$ in carbon nanotubes. The inset in figure $7 \mathrm{f}$ shows the SAED pattern of the InN nanotube with Bragg spot corresponding to the different planes of wurtzite InN. Optical absorption spectra of the nanowires and nanotubes gave a sharp band around $\sim 0.8 \mathrm{eV}$ [11]. Interestingly, no distinct absorption features were found in the $1-2 \mathrm{eV}$ region.

AlN, GaN and InN nanowires using urea-complexes as precursors: It is known that urea can act as a nitriding agent by generating $\mathrm{NH}_{3}$ in situ in reactions with metal ions $[20,21]$. We have characterized single-source precursors involving urea complexes with the metal chlorides, which produce nitride nanostructures at elevated temperatures in an inert atmosphere, or under solvothermal conditions [22]. Thus, the decomposition of $\left[\mathrm{M}\left(\mathrm{H}_{2} \mathrm{NCONH}_{2}\right)_{6}\right] \mathrm{Cl}_{3}(\mathrm{M}=\mathrm{Al}, \mathrm{Ga})$ on $\mathrm{Si}(100)$ substrates containing gold clusters, under a flow of nitrogen $(99.999 \%, \sim 200 \mathrm{sccm})$ gave good yields of AlN and GaN nanowires at 1000 and $800^{\circ} \mathrm{C}$ respectively. Decomposition of $\mathrm{In}\left(\mathrm{H}_{2} \mathrm{NCONH}_{2}\right)_{3} \mathrm{Cl}_{3}$ in $\mathrm{NH}_{3}$ gave $\mathrm{InN}$ nanowires at $550^{\circ} \mathrm{C}$. We show $\mathrm{SEM}$ images of the AlN, GaN and InN nanowires in figure $8 \mathrm{a}-\mathrm{c}$ respectively. All the nanowires have hexagonal structures and the nanowire diameter varies in the 20$100 \mathrm{~nm}$ range, with the lengths going up to a few microns. The lattice fringes in the HREM images in figure 8a-c reveal spacings of $2.66,2.57$ and $2.68 \AA$ corresponding to the (100), (002) and (101) planes of the wurtzite structures of AlN, GaN, and $\mathrm{InN}$ respectively. The PL spectrum of the GaN nanowires at room temperature shows band-edge luminescence centered around $370 \mathrm{~nm}(3.35 \mathrm{eV})$. The InN nanowires show an absorption band centered at $\sim 0.7 \mathrm{eV}$ which is now considered to be the intrinsic band gap of InN. We have employed the urea complexes to prepare nanocrystals of the metal nitrides as well.

Nebulized spray pyrolysis (NSP): Single crystalline GaN and InN nanowires could also be prepared by the reaction of acetylacetonates of Ga and In with $\mathrm{NH}_{3}$ on $\mathrm{Si}(100)$ substrates covered with catalytic amount of gold clusters, by nebulized spray pyrolysis (NSP) technique $[11,23]$. In this method, a dilute solution $(\sim 1$ $\mathrm{mM}$ ) of the metal acetylacetonate precursor in methanol solution is atomized using a PZT transducer that vibrates at $\sim 1.72 \mathrm{MHz}$. The atomized vapour is then carried out using $\mathrm{NH}_{3}$ as carrier gas and passed over the $\mathrm{Si}(100)$ substrates covered with gold particles inside a furnace which is preheated at desired temperature. GaN nanowires were grown in the $800-900^{\circ} \mathrm{C}$ range while $\mathrm{InN}$ nanowires around $580^{\circ} \mathrm{C}$. $\mathrm{NH}_{3}$ acts as the nitriding agent and the gold particles catalyze one-dimensional growth of the nanowires. We show typical SEM images of the nanowires in figures 9a and 9c, inset in figure 9a shows a typical low-magnification SEM image of the 

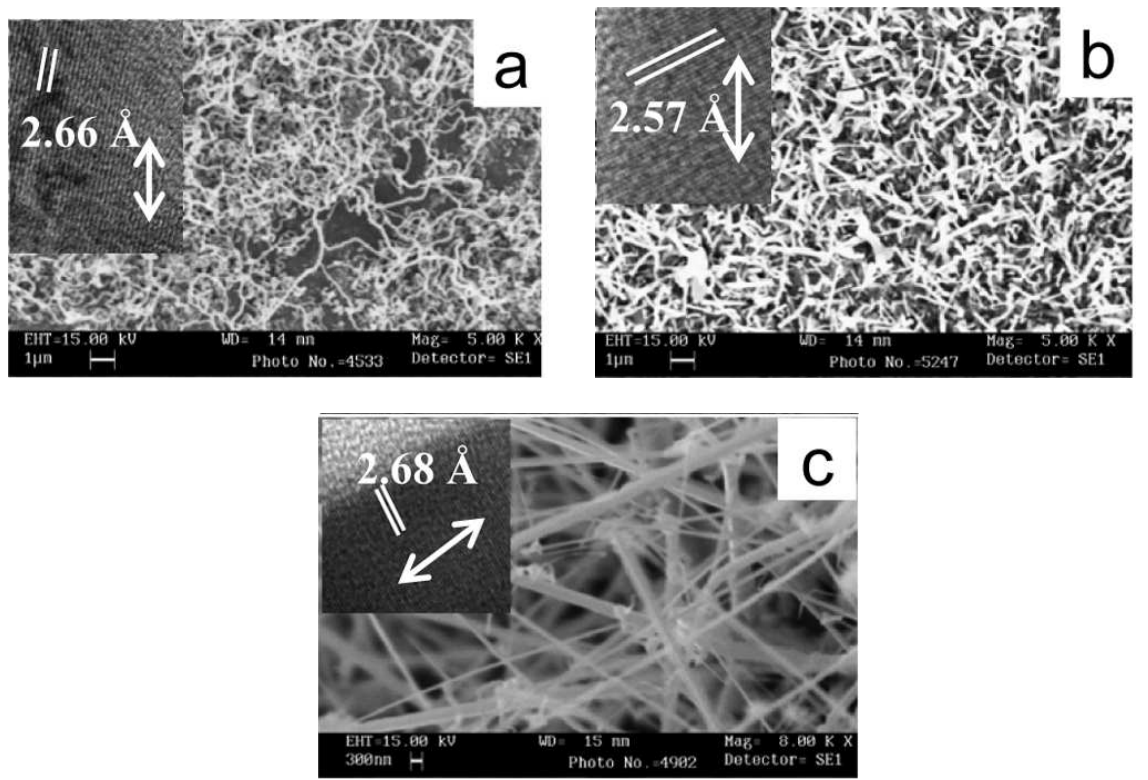

Figure 8. SEM images of (a) AlN, (b) GaN, (c) InN nanowires prepared by the decomposition of the respective urea precursors. Insets show HREM images (double headed arrows indicate the long axes of the crystal).

nanowires. The XRD pattern of the nanowires was characteristic of the hexagonal structure. The TEM images (insets in figures $9 \mathrm{~b}$ and $9 \mathrm{~d}$ ) show the nanowires to have diameters in the range of $10-50 \mathrm{~nm}$, and a few microns long. The nanowires are single crystalline as proved by the analysis of SAED patterns (insets in figures $9 \mathrm{~b}$ and $9 \mathrm{~d})$, with spots corresponding to the (100), (002), (101) planes of the wurtzite structure. The HREM images in figure $9 \mathrm{~b}$ and $9 \mathrm{~d}$ show lattice fringes with a spacing of $\sim 2.43$ and $2.70 \AA$, corresponding to the (101) planes of the wurtzite structure of $\mathrm{GaN}$ and $\mathrm{InN}$ respectively. The growth direction and the crystal long axis of the nanowires are in the $\langle 101\rangle$ direction. GaN nanowires show the characteristic emission band centered $\sim 370 \mathrm{~nm}$ in the PL spectrum. The optical absorption spectrum of the InN nanowires show a broad band in the 900-1200 nm region, and a sharp feature around $1575 \mathrm{~nm}(0.79 \mathrm{eV})$.

\section{Ultra-thin nanocrystalline films prepared by using the liquid-liquid interface}

The air-water interface has been traditionally employed to prepare particle assemblies and films of metals and semiconductors. The liquid-liquid interface between water and an organic liquid, however, has not been investigated sufficiently for possible use in preparing nanocrystals and thin films of materials. We demonstrate the use of the liquid-liquid interface as a medium for preparing ultra-thin films of metal 

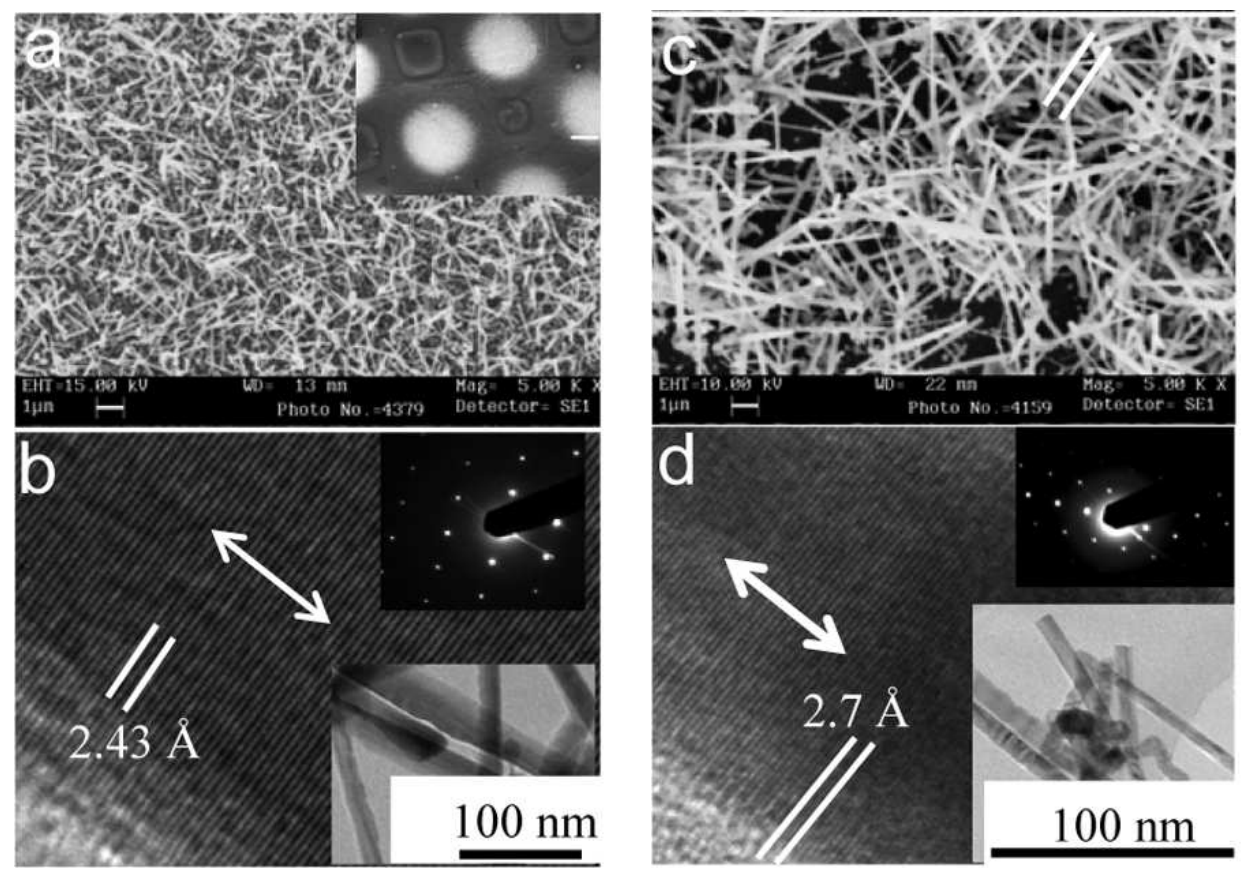

Figure 9. (a) and (c) SEM images of the GaN and InN nanowires on $\mathrm{Si}\left(\begin{array}{lll}1 & 0 & 0\end{array}\right)$ substrates. Inset in (a) is the low-magnification SEM image. (b) and (d) are HREM images of the GaN and InN nanowires respectively. Insets in (b) and (d) are low-magnification TEM images (bottom right) and SAED pattern on a nanowire (top right).

chalcogenides. The method involves the reaction at the interface between a metalorganic compound in the organic layer and an appropriate reagent for sulfidation in the aqueous layer.

\section{$4.1 C d S$}

To prepare CdS nanocrystals [24], cadmium cupferronate $\left(\mathrm{Cd}(\operatorname{cup})_{2}\right)$ was used as the cadmium source and $\mathrm{Na}_{2} \mathrm{~S}$ as the sulfur source. In a typical experiment, 0.0045 $\mathrm{g}$ of $\mathrm{Na}_{2} \mathrm{~S}$ was dissolved in $30 \mathrm{ml}$ of water $(2 \mathrm{mM})$ in a $100 \mathrm{ml}$ beaker and $0.0125 \mathrm{~g}$ of $\mathrm{Cd}(\mathrm{cup})_{2}$ dissolved in $30 \mathrm{ml}$ of toluene $(1 \mathrm{mM})$ by ultra-sonication. A few drops of n-octylamine were added to the $\mathrm{Cd}(\mathrm{cup})_{2}$ solution in order to make it completely soluble. The toluene solution was slowly added to a beaker containing the aqueous $\mathrm{Na}_{2} \mathrm{~S}$ solution. The interface started appearing yellow within a few minutes. The yellow film of CdS at the liquid-liquid interface can be seen in figure 10a. Note that both the liquid layers are colourless. The film was lifted with the help of a glass slide and was used for characterization. CdS nanocrystalline films could also be prepared by using cadmium stearate, $\mathrm{Cd}(\mathrm{st})_{2}$, as the metal source. In figure 10c, we show a typical TEM image of CdS nanocrystallites formed at the interface 

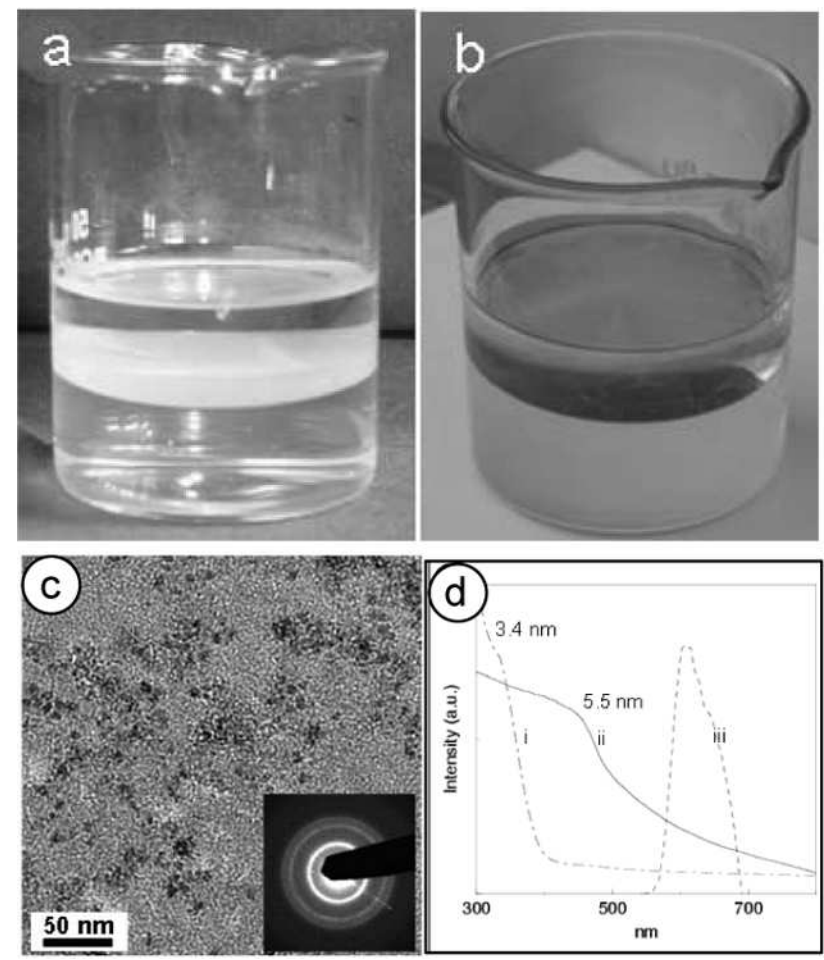

Figure 10. Nanocrystalline films of (a) CdS and (b) CuS formed at the water-toluene interface. (c) TEM image of $5.5 \mathrm{~nm}$ CdS nanocrystals obtained at room temperature at the interface (inset gives the SAED pattern). (d) UV-visible absorption spectra of (i) $3.5 \mathrm{~nm}$ and (ii) $5.5 \mathrm{~nm}$ CdS nanocrystals. (iii) Photoluminescence spectrum of $5.5 \mathrm{~nm}$ CdS nanocrystals.

obtained by the above-mentioned procedure. An excess of sulfide was required to completely react with the cupferronate. It can be seen that the film consists of monodisperse $5.5 \mathrm{~nm}$ nanocrystals which corresponds well with the particle size of $5 \mathrm{~nm}$ calculated from the X-ray peak broadening of the nanocrystals. The inset in figure 10c is the SAED pattern of the nanocrystals showing crystalline nature well. The size of the nanocrystals formed at the interface could be varied by controlling reaction conditions as well as the choice of solvent and the metal precursor. Thus, CdS nanocrystals of $6.5 \mathrm{~nm}$ diameter were obtained by carrying out this reaction at $75^{\circ} \mathrm{C}$. Increasing the concentration of the reactants also yielded bigger nanocrystals. The size of the nanocrystals could be reduced by controlling the diffusion rate of the $\mathrm{S}^{2-}$ ions. For example, nanocrystals of $3.5 \mathrm{~nm}$ diameter were obtained by doubling the viscosity of the aqueous medium by using a 24 mass\% glycerol-water mixture. Because of the higher viscosity of the aqueous medium, the diffusion of the $\mathrm{S}^{2-}$ ions to the interface decreases, leading to a slow reaction rate. CdS nanocrystals of $10 \mathrm{~nm}$ diameter were obtained when the reaction was carried out with $2.5 \mathrm{mM}$ of $\mathrm{Cd}(\mathrm{st})_{2}$ and $5.0 \mathrm{mM}$ of $\mathrm{Na}_{2} \mathrm{~S}$ for $12 \mathrm{~h}$. The UV-visible spectrum of the $5.5 \mathrm{~nm} \mathrm{CdS}$ nanocrystals (figure 10d) shows a broad absorption maximum around $\sim 450 \mathrm{~nm}$ 


\section{Ujjal K Gautam et al}

which is blue-shifted compared to the bulk CdS (absorption maximum at $515 \mathrm{~nm}$ ) due to quantum confinement. The $3.5 \mathrm{~nm}$ nanocrystals show the band at $\sim 340 \mathrm{~nm}$. The photoluminescence spectrum of the $5.5 \mathrm{~nm}$ particles shows a peak at $610 \mathrm{~nm}$. We have reported nanocrystals of other materials such as CoS, NiS and ZnS as well by employing the same method [25].

\section{$4.2 C u S$ and $C u S e$}

We have been able to obtain ultra-thin single-crystalline films of $\mathrm{CuS}$ and $\mathrm{CuSe}$ using the liquid-liquid interface [26]. In a typical preparation of $\mathrm{CuS}$ films, 75 $\mathrm{ml}$ of $0.12 \mathrm{mM} \mathrm{Cu}(\mathrm{cup})_{2}$ solution in toluene was added slowly to $75 \mathrm{ml} 0.5 \mathrm{mM}$ of $\mathrm{Na}_{2} \mathrm{~S}$ taken in a crystallization dish (10 cm diameter). An excess of $\mathrm{Na}_{2} \mathrm{~S}$ was required in order to prevent the formation of $\mathrm{Cu}_{2} \mathrm{~S}$. The interface gradually turns green and the $\mathrm{CuS}$ film is formed at the interface after $12 \mathrm{~h}$, while the two liquid phases remained colourless (figure 10b). In figure 11a we show a TEM image of such a film formed at the interface. The film is single-crystalline as revealed by

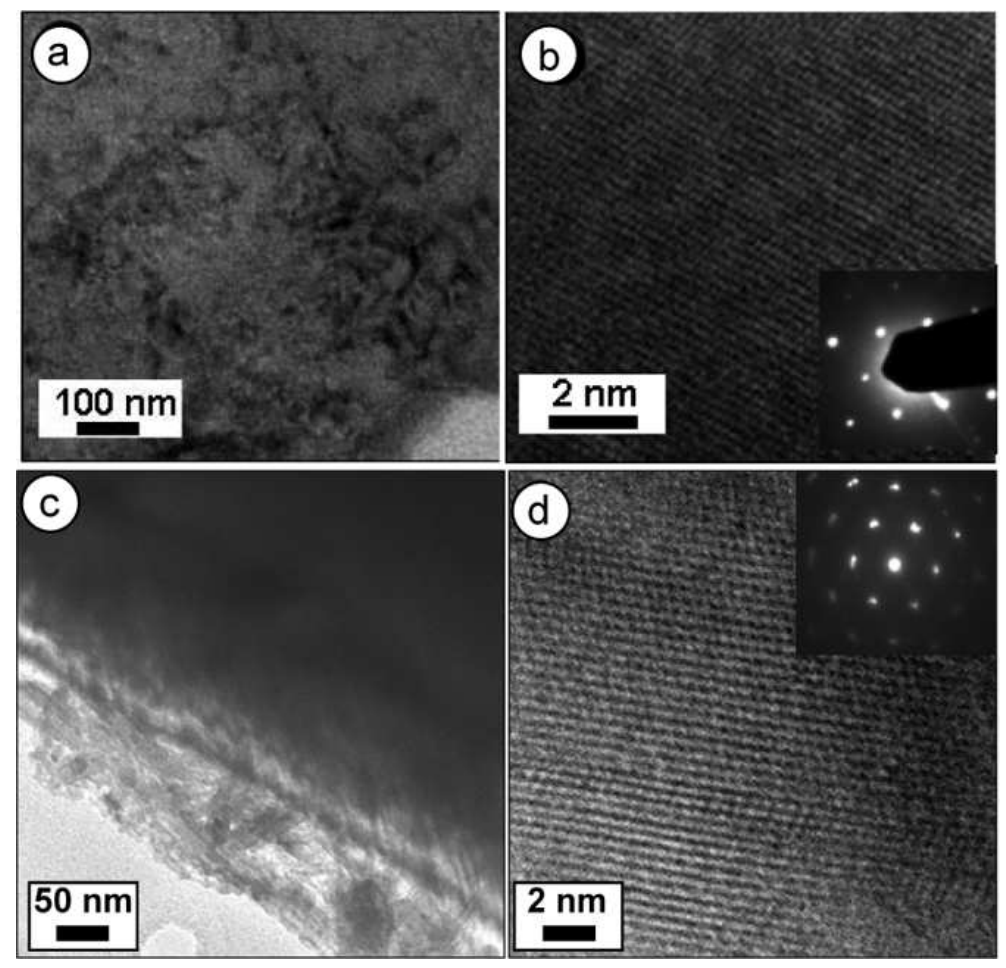

Figure 11. (a) TEM image of CuS films obtained by using $0.09 \mathrm{mM}$ of $\mathrm{Cu}(\text { cup })_{2}$ after $12 \mathrm{~h}$. (b) HREM image of the film (inset is the corresponding SAED pattern). (c) TEM image of a CuSe ultra-thin film formed at the toluene-water interface. (d) HREM image of the film. Inset shows the corresponding SAED pattern. 


\section{Soft chemical routes to semiconductor nanostructures}

the HREM image in figure 11b. The lattice spacing of $2.7 \AA$ in the HREM image corresponds to the separation between (006) planes of the hexagonal $\mathrm{CuS}$ phase. The inset in figure 11b shows the corresponding SAED pattern indicating the singlecrystalline nature of the film. The diffraction spots could be indexed on the basis of the hexagonal structure ( $\mathrm{P} 6_{3} / \mathrm{mmc}, a=3.792 \AA$ and $c=16.34 \AA$, JCPDF no. 06-0464). The thickness of the films was generally less than $100 \mathrm{~nm}$, the lateral dimensions extending over several microns. The thickness of the above-mentioned film was estimated from the AFM images and by ellipsometry to be in the range of 40-60 nm. The film grows slowly with time, first appearing as green islands at the interface (in the initial 1-2 h) and slowly covering the entire interface. The nature of the film could be varied by varying reaction conditions. For example, using a lower reactant concentration, we obtained thinner films, and by carrying out the reaction at higher temperature, we obtained highly crystalline smaller film fragments.

We prepared CuSe ultra-thin nanocrystalline films at the interface by reacting $\mathrm{Cu}(\text { cup })_{2}$ in the organic layer with $\mathrm{Na}_{2} \mathrm{Se}$ in the aqueous layer. In figure 11c, we show a typical TEM image of a CuSe film obtained at room temperature by using $75 \mathrm{ml}$ of $0.12 \mathrm{mM} \mathrm{Cu}(\text { cup })_{2}$ solution in toluene and $75 \mathrm{ml} 0.5 \mathrm{mM}$ of $\mathrm{Na}_{2}$ Se solution in a $10 \mathrm{~cm}$ diameter reaction vessel. The film is single-crystalline with a thickness of $\sim 30 \mathrm{~nm}$ extending over several microns. The HREM image of the film in figure 11d shows lattice planes separated by $3.5 \AA$ corresponding to the (100) planes of the hexagonal CuSe phase. The inset in figure 11d shows the SAED pattern confirming the single crystalline nature of the film.

\section{Conclusions}

The above discussion should suffice to demonstrate the success of some of the softchemical routes in preparing nanocrystals, nanowires and related structures of semiconducting metal chalcogenides and nitrides. In particular, the use of new reagents and precursors has yielded excellent results. What is interesting is the simplicity of the synthesis and good quality of the nanocrystals in the soft-chemical methods. It is noteworthy that CdS and CdSe nanowires and nanotubes have been obtained by surfactant-assisted synthesis [27]. Recently, $\mathrm{Fe}_{1-x} \mathrm{~S}$ nanowires were prepared by the low-temperature decomposition of an addition compound of $\mathrm{Fe}_{1-x} \mathrm{~S}$ with ethylene diamine [28]. Similarly, organo-ammonium precursors were employed to prepare nanowires of $\mathrm{GeS}_{2}$ and $\mathrm{GeSe}_{2}$ [29]. The potential of the liquid-liquid interface in generating ultra-thin films of semiconducting materials is specially noteworthy.

\section{References}

[1] The chemistry of nanomaterials edited by C N R Rao, A Mueller and A K Cheetham (Wiley-VCH, Weinheim, 2004)

[2] C N R Rao, F L Deepak, G Gundiah and A Govindaraj, Progress in Solid State Chemistry 31, 5 (2003)

[3] Z L Wang, Adv. Mater. 15, 351 (2003) 


\section{Ujjal K Gautam et al}

[4] R Tenne and C N R Rao, Philosophical Transactions: Mathematical, Physical and Engineering Sciences 362, 2099 (2004)

[5] C N R Rao and M Nath, Dalton Trans. 1 (2003)

[6] U K Gautam, R Seshadri and C N R Rao, Chem. Phys. Lett. 375, 560 (2003)

[7] L Spanhel, H Haase, H Weller and A Henglein, J. Am. Chem. Soc. 109, 5649 (1987)

[8] U K Gautam, M Rajamathi, F Meldrum, P Morgan and R Seshadri, Chem. Commun. $629(2001)$

[9] U K Gautam and R Seshadri, Mater. Res. Bull. 39, 669 (2004)

[10] K Sardar and C N R Rao, Adv. Mater. 16, 425 (2004)

[11] K Sardar, F L Deepak, Md M Seikh, A Govindaraj and C N R Rao, Small 1, 91 (2005)

[12] K Sardar and C N R Rao, Solid State Sci. 7, 217 (2005)

[13] K E Gonsalves, S P Rangarajan and G Carlson, Appl. Phys. Lett. 71, 2175 (1997)

[14] J Wu, W Walukiewicz, K M Yu, J W Ager III, E E Haller, H Lu, W J Schaff, Y Saito and Y Nanishi, Appl. Phys. Lett. 80, 3967 (2002)

[15] F Bechstedt and J Furthermuller, J. Crystal Growth 246, 315 (2002)

[16] Selenium edited by R A Zingaro and W C Cooper (Van Nostrand Reinhold, New York, 1974)

[17] U K Gautam, M Nath and C N R Rao, J. Mater. Chem. 13, 2845 (2003)

[18] D L Klayman and T S Griffin, J. Am. Chem. Soc. 95, 197 (1973)

[19] U K Gautam and C N R Rao, J. Mater. Chem. 14, 2530 (2004)

[20] S Podsiadlo, Thermochem. Acta 256, 367 (1995)

[21] Y Qiu and L Gao, J. Am. Ceram. Soc. 87, 352 (2004)

[22] K Sardar, M Dan, B Schwenzer and C N R Rao, J. Mater. Chem. 22, 2175 (2005)

[23] S R C Vivekchand, G Gundiah, A Govindaraj and C N R Rao, Adv. Mater. 16, 1842 (2004)

[24] U K Gautam, M Ghosh and C N R Rao, Chem. Phys. Lett. 381, 1 (2003)

[25] C N R Rao, G U Kulkarni, V V Agarwal, U K Gautam and M Ghosh, J. Colloid Interface Sci. 289, 305 (2005)

[26] U K Gautam, M Ghosh and C N R Rao, Langmuir 20, 10775 (2004)

[27] C N R Rao, A Govindaraj, F Leonard Deepak, N A Gunari and M Nath, Appl. Phys. Lett. 78, 1853 (2001)

[28] M. Nath, A Choudhury, A Kundu and C N R Rao, Adv. Mater. 15, 2098 (2003)

[29] M Nath, A Choudhury and C N R Rao, Chem. Commun. 2698 (2004) 